\title{
Colorimetric properties of the light scattered by various objects
}

\author{
Michel Lequime, Laetitia Abel and Carole Deumié \\ Institut FRESNEL - UMR CNRS 6133 \\ Domaine Universitaire de Saint-Jérôme, 13397 Marseille Cedex 20 - France
}

\begin{abstract}
The artificial reproduction of some coloration effects (for instance nacre aspect, iridescence) which appear spontaneously in the nature needs a right description of the different mechanisms which are involved in these phenomena and especially, a detailed analysis of the spectral behavior of the light scattered by such surfaces. With this aim in sight, we have developed a dedicated set-up for the recording of the reflectance distribution function of solid samples in the whole visible spectral range. We have also analyzed the different methods which are able to describe the color information for scattered light. First experimental results obtained on some organic glass windows are given in conclusion.
\end{abstract}

Keywords: Scattered Light, Colorimetry

\section{INTRODUCTION}

The characterization of the scattering properties of an optical element like a window or a plane mirror needs the use of a dedicated measurement set-up which is able to record the angular distribution of the light transmitted or reflected by this element when it is illuminated by a well collimated beam. This set-up, often called ARS set-up (for Angle Resolved Scattering set-up) includes mainly a powerful light source with a low divergence, like a laser, and a small size photodetector located on a rotating arm [1].

It is naturally possible to perform in the recording of this angular distribution function with several laser lines, and to compare the results obtained at these few discrete wavelengths [2]. But, concerning the characterizing of the scattering properties of an optical element in the whole visible range, this kind of solution has two main drawbacks: first, the covering of the spectral range is not homogeneous, and secondly the acquisition time becomes prohibitive. The use of a tunable dye laser can provide with a part of a solution, but the complexity of the set-up is greatly enhanced.

If we aim at recording the BRDF quantity (Bidirectional Reflectance Distribution Function) in the whole visible range, and being in the same time in accordance with the standards defined by the International Commission on Illumination (CIE), we need to develop a new experimental set-up which satisfies the following specifications:

- Measurement spectral range : from $380 \mathrm{~nm}$ up to $780 \mathrm{~nm}$

- Spectral interval : $5 \mathrm{~nm}$

- Angular resolution : better than 1 degree

- Overall acquisition time : less than 10 minutes

- Dynamic range : more than $10^{9}$

The two first specifications are in accordance with the CIE guidance, while the third one aims only at providing with accurate data. The dynamic range specification allows considering the recording with the same set-up of the transmitted, reflected and scattered light beams.

\section{DESCRIPTION OF THE COLORIMETRIC ARS SET-UP}

This new Angle Resolved Scattering set-up with colorimetric features includes mainly 4 items, i.e. a Light Source, a Lightning Assembly, a Detection Assembly and a Data Processing System (see Figure 1).

The Light Source is a 7 Watts quartz halogen lamp, with a continuous emission spectrum from $360 \mathrm{~nm}$ up to $2 \mu \mathrm{m}$. The light power delivered by this source in the visible range is launched into a silica step index multimode fiber called $\mathbf{A}$ (core diameter 200 microns, numerical aperture 0.25). 
The extremity of this A fiber is located at the object plane of an achromatic lens (focal length about $50 \mathrm{~mm}$ ) in order to obtain a low divergence light beam. This collimated beam crosses a filter wheel, which holds four different optical densities, respectively equal to 0 (no density in the filter holder), 2, 4, and $\infty$ (in this case, the density is simply a stop). The use of such sub-assembly has two main goals: first, the adjustment in a large range $\left(1\right.$ to $\left.10^{-4}\right)$ of the amount of light provided by the source in order to optimize the detection level and second, the possibility to record for each measurement the dark response of the whole set-up.

The transmitted light beam is then focused with the same achromatic lens on the extremity of a new multimode fiber, called B and identical to the previous one.

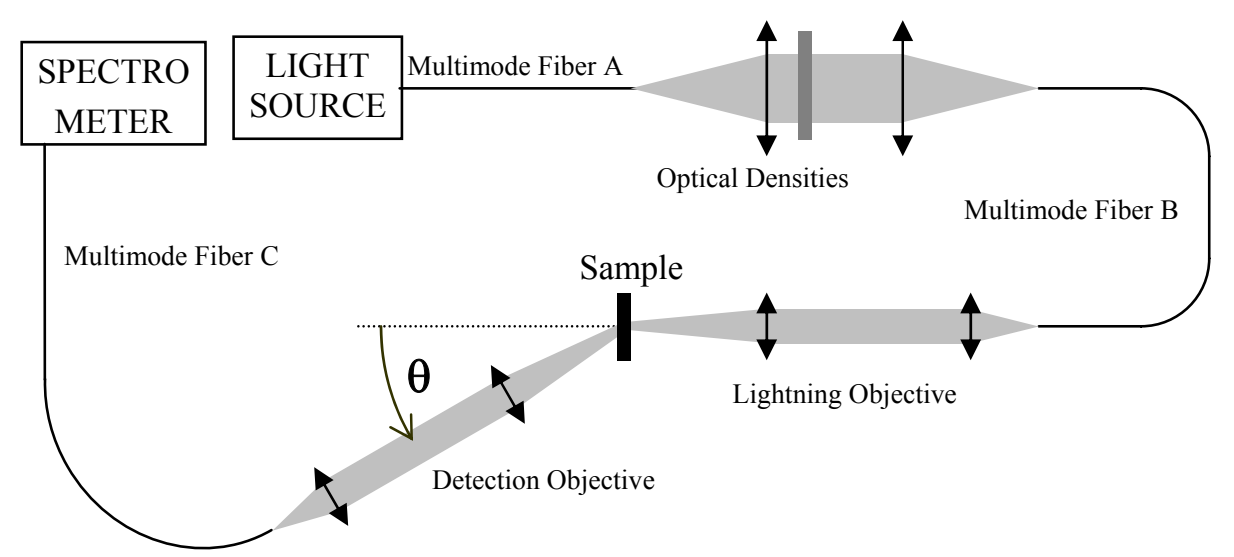

Figure 1 - Schematic representation of our colorimetric ARS set-up

The second extremity of this B fiber is located at the object focal plane of an achromatic lens (focal length about 10 $\mathrm{mm}$ ), followed by a second achromatic lens (focal length about $190 \mathrm{~mm}$ ), the distance between both lenses being equal to the sum of their focal lengths (i.e. $200 \mathrm{~mm}$ ). Accordingly, the image of the fiber provided by this lightning objective is located in the image focal plane of the second achromat, and its diameter is equal to $3.8 \mathrm{~mm}$ (the magnification of this lightning objective is indeed equal to the ratio of the focal distances of both achromats, i.e. about 19). In the same time, the divergence of the output beam is reduced to less than 1 degree (the numerical aperture of the fiber divided by the magnification of the lightning objective).

Besides, the far field pattern of the B fiber is imaged in the focal plane of the first achromatic lens, which coincides moreover with the object focal plane of the second one: this configuration is a telecentric one, and ensures that the illumination conditions of the sample are perfectly stable into the illuminated area, if this sample is located in the close vicinity of the plane corresponding to the image of the fiber (for each point inside this illuminated area, the mean ray is always perpendicular to the sample, while the angular divergence of the lightning beam remains constant).

The light power transmitted or scattered by the sample installed in this particular configuration is now collected by a detection objective, identical to the lighting objective, but used in a symmetric way (see Figure 1). This second objective is installed on a rotating arm and the rotation axis of this mobile arm belongs to the input plane of the sample (which is also the image plane of the lightning objective and the object plane of the detection objective).

When both objectives are perfectly aligned, the whole system provides with a final image with the same optical features as the B fiber, and which can be coupled with a very high efficiency (close to $100 \%$ ) to a $\mathbf{C}$ fiber identical to the B one. When the axis of the detection objective is rotated by an angle $\theta$, the system allows recording the light scattered by the sample at this specific angle, with the same intrinsic efficiency (the image of the fiber seeing under an angle $\theta$ is always smaller than the fiber itself). Moreover, this configuration provides with a very high rejection of the parasitic light, since the optical throughput of the detection assembly (detection objective plus detection fiber) is in practice restricted to the recording of the light transmitted or scattered by the sample: this detection configuration is very similar to a confocal one. 
The polychromatic light power collected by the $\mathrm{C}$ fiber is launched into a miniature USB2000 OCEAN OPTICS spectrometer, with the following features:

- Input slit: 100 microns width

- Diffraction grating: 600 lines $/ \mathrm{mm}$, blazed at $500 \mathrm{~nm}$

- Cylindrical miniature lens (used to increase the detection efficiency by focusing the light beam in the direction perpendicular to the linear CCD array)

- 2048 elements linear silicon CCD array (SONY ILX511) with order sorting coating directly deposited on the cover glass of the detector

- Integrated 12 bits A/D converter

- Spectral resolution: $5 \mathrm{~nm}$

- Integration time adjustable by software from $3 \mathrm{~ms}$ up to $3 \mathrm{~s}$

The whole set-up is completely computer controlled: driving of the filter wheel, automatic definition of the CCD integration time, in order to optimize the signal to noise ratio on the detector and to avoid any saturation effects, recording of the dark response of the $\mathrm{CCD}$ for the selected integration time. The same PC is used to perform the recording and the processing of the data, which can be shared in two main levels:

- a low level processing, which includes the recording of the digital information provided by the spectrometer (data exchange through the USB port), the subtraction of the dark signals, the storage of all the spectral data for each detection angle and the use of the calibration data to provide with absolute and accurate measurements,

- a high level processing, which is dedicated to a data reduction process. Indeed, the number of digital information associated to the characterization of the scattering properties of a given sample is quite huge, in the range of 100000 (typically 50 different angles with $2000 \mathrm{CCD}$ pixels data for each angle). To allow an easy comparison of the spectral scattering properties of various samples, it is required to identify a simple way to synthesize all these experimental results. The selected method is based on the rules defined by the CIE for the determination of the color properties of an object [3].

If we note $s(\theta, k)$ the low level processed signal associated to the angle $\theta$ and pixel $k$, we perform first a sampling of this signal each $5 \mathrm{~nm}$ between 380 and $780 \mathrm{~nm}$ at fixed angle (it requires naturally the knowledge of the spectral calibration curve of the CCD array). This $5 \mathrm{~nm}$ sampling interval is in accordance with the CIE rules and also with the effective spectral resolution of our miniature spectrometer. The resulting information is noted $S(\theta, \lambda)$. Then by using the three eye response curves $x(\lambda), y(\lambda)$ and $z(\lambda)$ represented at the Figure 2, we can compute three new functions noted $X(\theta), Y(\theta)$ and $Z(\theta)$, and defined by

$$
X(\theta)=\frac{\sum x(\lambda) S(\theta, \lambda)}{\sum x(\lambda)} \quad Y(\theta)=\frac{\sum y(\lambda) S(\theta, \lambda)}{\sum y(\lambda)} \quad Z(\theta)=\frac{\sum z(\lambda) S(\theta, \lambda)}{\sum z(\lambda)}
$$

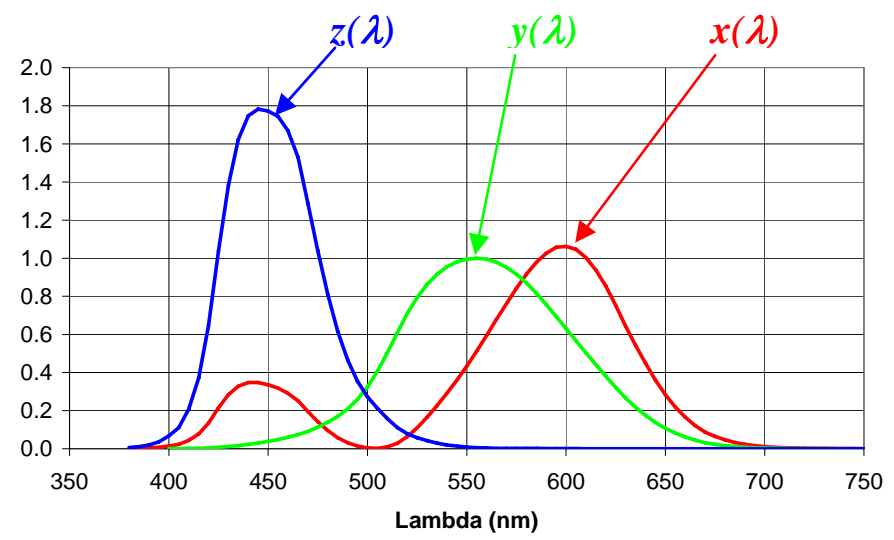

Figure 2 - Eye response curves 
The number of data is then reduced to about 150 (50 different angles with 3 colorimetric data for each angle). Naturally, the dependence of these scattering functions with respect to the angle is very strong and can mask the color information. Therefore the use of ratios seems a good way to overcome this difficulty. It is the reason why we have chosen to replace the three color stimulus $X(\theta), Y(\theta)$ and $Z(\theta)$ by the alternative equivalent set $Y(\theta), x(\theta)$ and $y(\theta)$, the two last functions being defined by

$$
x(\theta)=\frac{X(\theta)}{X(\theta)+Y(\theta)+Z(\theta)} \quad y(\theta)=\frac{Y(\theta)}{X(\theta)+Y(\theta)+Z(\theta)}
$$

Thus the colorimetric properties of the light scattered by a sample can be described by only three angular functions, the first one describing the evolution of the level of the scattered light with respect to the detection angle and the two others the coloration changes of the scattered light with respect to the same angle.

\section{QUALIFICATION OF THE COLORIMETRIC ARS SET-UP}

Our first qualification activity has been devoted to a measurement of the angular features of this new set-up and to an estimation of the dynamic range of the detection. It consists on a recording of the angular response of the set-up, at a single wavelength $(570 \mathrm{~nm})$ with and without sample. The experimental results are presented at the Figure 3.

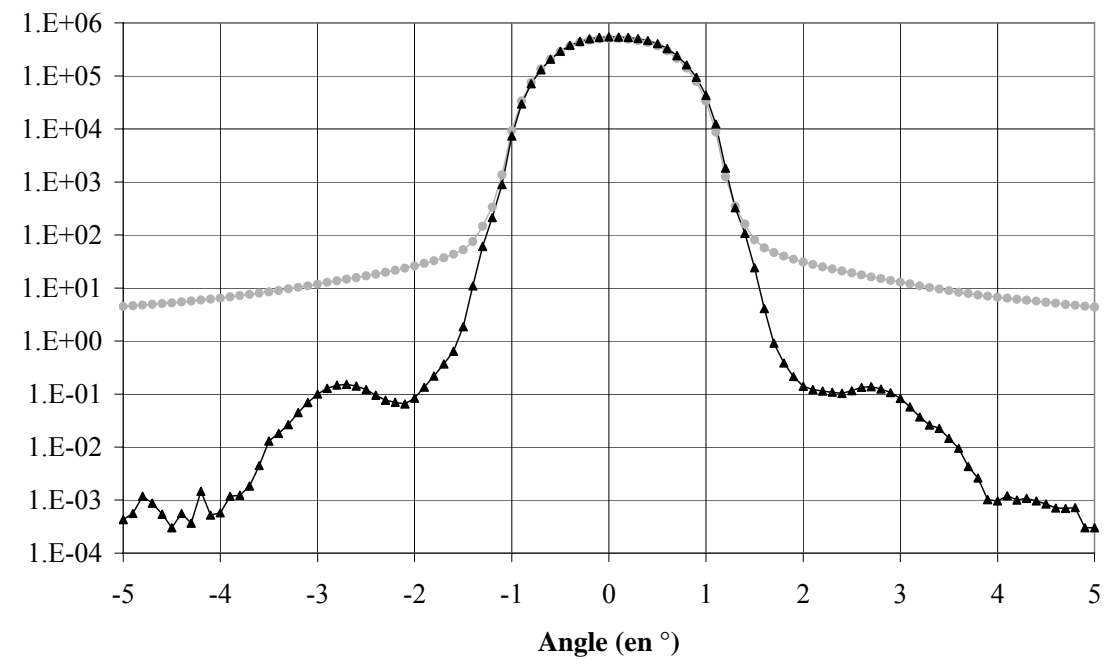

Figure 3 - Angular response of the set-up (with and without sample)

The black line curve (triangle dots) corresponds to the angular response of the set-up without sample: it shows that the divergence of the illumination beam ( 0.75 degrees HWHM) and the dynamic range $\left(10^{9}\right)$ are in good accordance with the expected values. The grey line curve (circular dots) has been recorded with an organic glass plate mounted into the sample holder, the angle of incidence of the illumination beam being equal to zero. We observe the same kind of behavior as before until 1.5 degree, where occurs a separation of the two curves, which allows to identify the own contribution of the sample: the ratio obtained at 2 degrees (more than 100) is sufficient to ensure an accurate determination of the scattering properties of such sample above this critical angle.

The second qualification activity concerns the calibration of our device with the help of a perfect diffuser. This recording is performed in reflection. It is important to stress here that our set-up is able to record the light scattered by a sample both in transmission and reflection.

The experimental results recorded at $630 \mathrm{~nm}$ are presented at the Figure 4, in linear and logarithmic scales, and compared first to the data obtained on the same item with a classical He-Ne ARS set-up, and second with the theoretical response associated to a perfect diffuser

$$
\operatorname{BRDF}(\theta) \cdot \cos \theta=(\rho / \pi) \cos \theta
$$



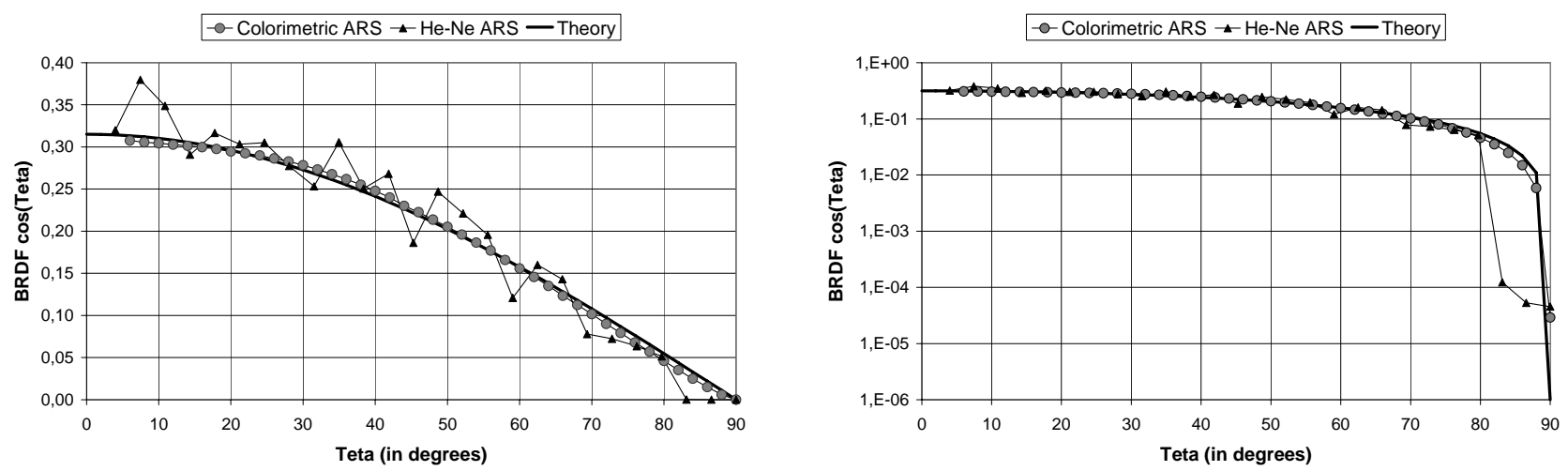

Figure 4 - Calibration curves obtained on a perfect diffuser (left curve: linear scale - right curve: log scale)

We can notice the smooth aspect of the data provided by our set-up (compared to the standard He-Ne ARS ones) and their perfect accordance with a theoretical modeling. The quality of our data is directly connected to the temporal incoherence of our light source and to the gentle angular averaging (about 1 degree) provided by the lightning and detection optical assembly.

Our last qualification activity is similar to the previous one, but the perfect diffuser is now replaced by a standard mirror. The comparison of the experimental results obtained with our colorimetric ARS set-up at $630 \mathrm{~nm}$ and with a standard $\mathrm{He}-\mathrm{Ne}$ one is achieved at the Figure 5.

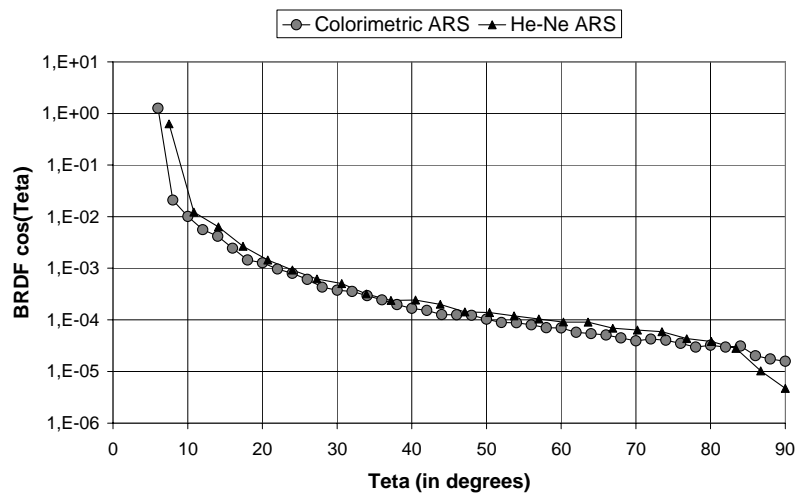

Figure 5 - BRDF data recorded on a standard mirror

Again, we can stress the regularity of the scattering function recorder by the colorimetric ARS set-up and the coherence of the absolute values provided by both set-ups.

\section{APPLICATION AREA AND PRELIMINARY RESULTS}

This colorimetric ARS set-up can be used for the characterization of the diffuse optical properties of various objects, like colored powders [4], pigments or coated spheres [5]. It can be also applied to the analysis of the roughness properties of transparent materials and to the identification of very small behavior difference, difficult to detect otherwise. The following experimental results have been obtained on some transparent windows ( $2 \mathrm{~mm}$ thick).

The curves presented at the Figure 6 shows, for the transmitted light

- the variation of the $Y(\theta)$ function with respect to the angle, between 0 and 90 degrees (this information will be now called Visual Scattering), 
- the variation of the color coordinates $x(\theta)$ and $y(\theta)$, again with respect to the angle but only between 0 and 80 degrees (and not 90 degrees), to avoid practical problems induced by the computation, at high angle, of the ratio between too noising data (this information will be henceforth called Colorimetric Scattering)
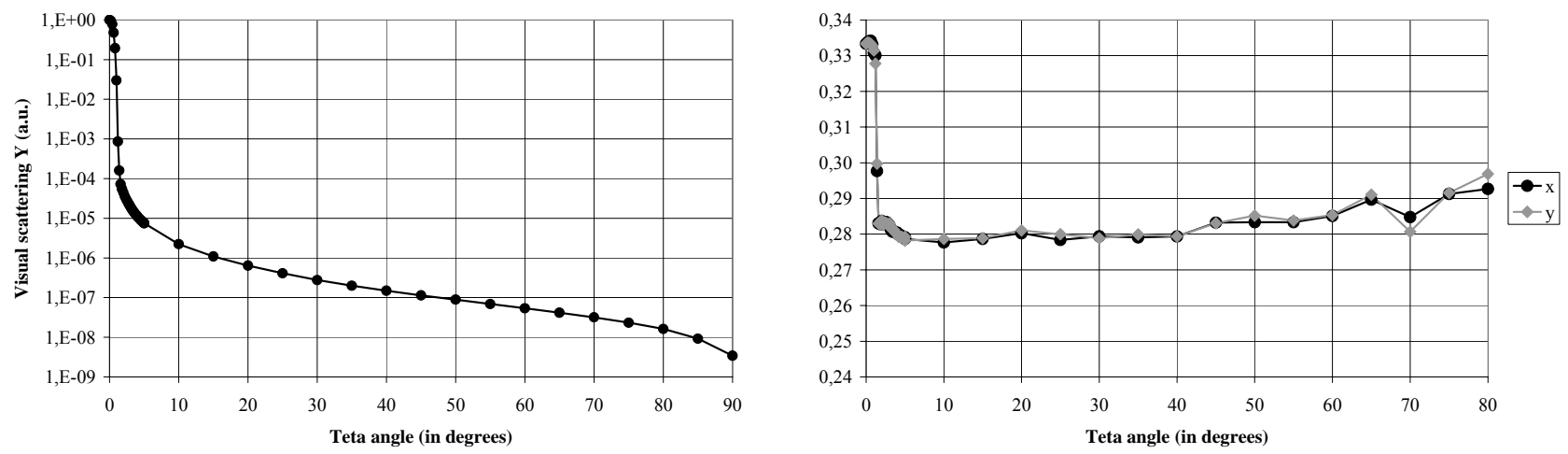

Figure 6 - Colorimetric properties of the light scattered by a transparent window (Material 1 - left curve: Visual Scattering - right curve: Colorimetric Scattering)

Let us stress the quite constant value obtained on this sample for the colorimetric scattering (about 0.28 between 5 and 60 degrees).

For a different sample, we can obtain completely different behavior, as presented at the Figure 7: the colorimetric scattering coordinates decrease rapidly at low angle down to 0.24 , then increases slowly up to 0.30 between 10 and 60 degrees.
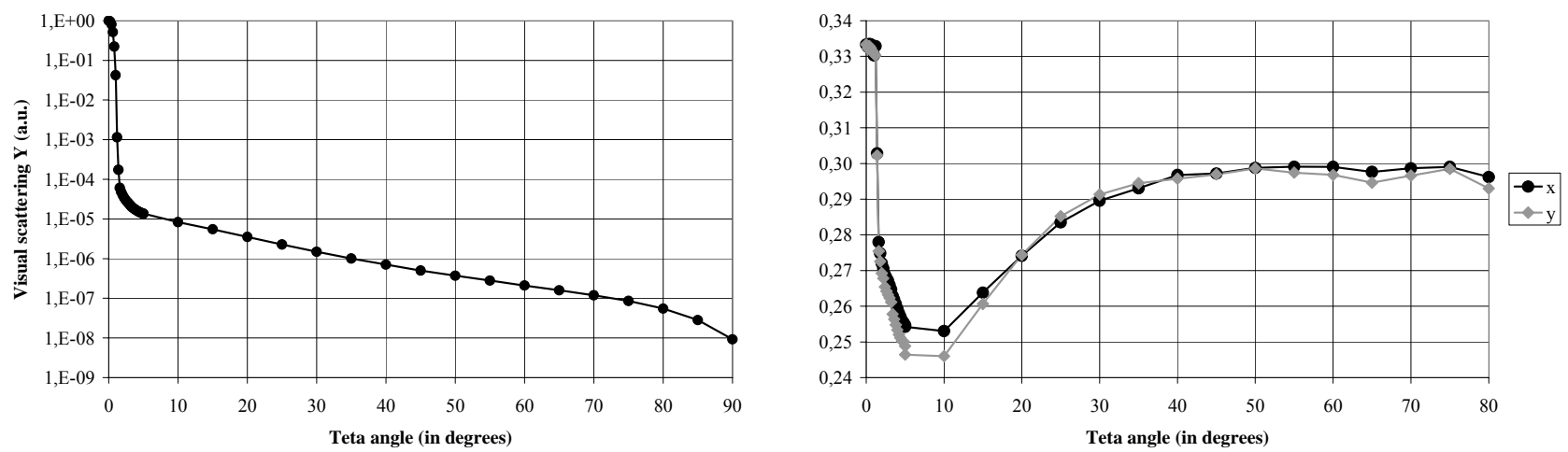

Figure 7 - Colorimetric properties of the light scattered by a second transparent window (Material 2 - left curve: Visual Scattering - right curve: Colorimetric Scattering)

Preliminary theoretical analysis seems to establish that the constant behavior of the colorimetric coordinates would be connected with the Gaussian part of the roughness spectrum (small correlation length), while the rapid increase between 10 and 60 degrees would be related to the Lorentzian part of the same roughness spectrum (large correlation length). Complementary analyses (both theoretical and experimental) have to been done to confirm (or reject) this very interesting hypothesis.

\section{CONCLUSION}

We have described the main features of an ARS set-up optimised for the recording of the scattering properties of various objects in the visible range, and for the analysis of the evolution of their colour properties with respect to this scattering angle. It can be applied to the study of coloured powders and coated spheres, but it can be also used for the characterisation of the roughness properties of transparent windows. The dynamic range of this new set-up can be 
improved by replacing the quartz halogen lamp by a low power xenon arc lamp ( 75 Watts for instance): this modification could allow recording the BRDF function of super polished substrates.

\section{REFERENCES}

1. C. Amra, Light scattering from multilayer optics - Part B: Application to experiment, J. Opt. Soc. Am. A, 11, 211$226(1994)$

2. C. Amra, D. Torricini and P. Roche, Multiwavelength $(0.45-10.6 \mathrm{~mm})$ angle-resolved scatterometer or how to extend the optical window, Appl. Opt., 32, 5462-5474 (1993)

3. see for instance http://www.cie.co.at/cie/ or http://cvision.ucsd.edu/cie.htm

4. C. Deumié, N. Destouches, M. Cathelinaud, G. Albrand, C. Cassagne and C. Amra, Optical Materials in powder forms : characterization techniques, Proceedings of SPIE 3738, 417-425 (1999)

5. C. Deumié, Ph. Voarino and C. Amra, Interferential powders for the spectral control of light scattering, SPIE Conference Advances in Optical Thin Films, Saint-Etienne (2003) 